H. O. RUCKER, W. S. KURTH, P. LOUARN, and G. FISCHER (Eds.)

PLANETARY RADIO EMISSIONS VII 


\section{Workshop Organizing Committee:}

Helmut O. Rucker

Space Research Institute

Austrian Academy of Sciences

and

Karl-Franzens University of Graz

Graz, Austria

William S. Kurth

Department of Physics and Astronomy

University of Iowa

Iowa City, Iowa, USA

Philippe Louarn

Centre d'Etude Spatiale de Rayonnements

CNRS/Université Paul-Sabatier Toulouse III

Toulouse, France

Georg Fischer

Space Research Institute

Austrian Academy of Sciences

Graz, Austria

Editorial/production, supervision

and interior design:

Manuel Scherf

Florian Topf 
H. O. RUCKER, W. S. KURTH, P. LOUARN, and G. FISCHER (Eds.)

\title{
PLANETARY RADIO EMISSIONS VII
}

\author{
Proceedings of the 7Th International Workshop on \\ Planetary, Solar and Heliospheric Radio Emissions \\ held at Graz, Austria
}

September $15-17,2010$

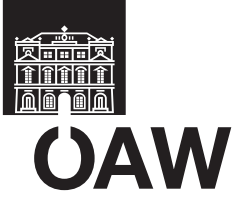

VERLAG DER

ÖSTERREICHISCHEN AKADEMIE DER WISSENSCHAFTEN

A-1010 WIEN, DR. IGNAZ SEIPEL-PLATZ 2 
Alle Rechte vorbehalten

ISBN 978-3-7001-7125-6

Copyright (C) 2011 by

Österreichische Akademie der Wissenschaften, Wien

Druck: Ferdinand Berger \& Söhne

Wiener Straße 80, 3580 Horn 